\title{
Tunable Entanglement Sudden Death and Birth in Cavity QED with Nonlinear Kerr-Like Medium
}

\author{
Q.-L. HE AND J.-B. XU* \\ Zhejiang Institute of Modern Physics and Physics Department \\ Zhejiang University, Hangzhou 310027, People's Republic of China \\ (Received January 6, 2011; revised version April 11, 2011; in final form May 16, 2011)
}

\begin{abstract}
The influence of nonlinear Kerr-like medium on the phenomenon of entanglement sudden death and birth in a double Jaynes-Cummings model is investigated. It is shown that the entanglement sudden death and birth phenomenon may appear in this system and the duration of entanglement sudden death and birth can be controlled by the nonlinear Kerr-like medium. Particularly, the phenomenon of entanglement sudden death and birth disappears one after another if we increase the value of the Kerr coefficient $\chi$ continuously, and all disappears if the value of the Kerr coefficient $\chi$ is large enough.
\end{abstract}

PACS: 03.65.Ud, 03.67.-a, 03.67.Mn

\section{Introduction}

It is well known that the entanglement is a special quantum correlation and has been viewed as an indispensable resource in quantum information processing [1-5]. The cavity quantum electrodynamics system offers a useful tool to generate entangled states [6] and manipulate entanglement. It can be used to create the entanglement between atoms in cavities and establish quantum communications between different optical cavities [7-9].

Control of the evolution of the qubit entanglement is one of the challenges of realizing quantum information processing. The entanglement dynamics of qubit pairs have attracted much attention [10-18] since $\mathrm{Yu}$ and Eberly [10] pointed out that the entanglement between two qubits interacting with uncorrelated reservoirs may disappear within a finite time during the dynamics evolution. This phenomenon, called entanglement sudden death (ESD) has been observed in the experiments $[19,20]$. Recently, the quantum dynamics of a system consisting of two cavities interacting with two independent reservoirs has been studied [21]. It is shown that ESD in a bipartite system independently coupled to reservoirs is related to the entanglement sudden birth (ESB) $[15,16]$ and the ESB could occur after, together, or even before ESD.

In this paper, we investigate the entanglement dynamics of a double two-photon Jaynes-Cummings model with an added nonlinear Kerr-like medium. Initially, the two atoms are prepared in the extended Werner-like states and the cavity field is prepared in the vacuum state. It is shown that the ESD and ESB phenomenon may appear in this system and the duration of ESD and ESB can

* corresponding author; e-mail: xujb@zju.edu.cn be altered by the nonlinear Kerr-like medium. In addition, the amount of the entanglement of the atoms or the other bipartite partitions of the system can be obviously altered by applying nonlinear Kerr-like medium. Particularly, the phenomenon of ESD and ESB disappears one after another if we increase the value of the Kerr coefficient $\chi$ continuously, and all disappears if the value of the Kerr coefficient $\chi$ is large enough.

\section{The influence of nonlinear Kerr-like medium on the entanglement sudden death and sudden birth}

We consider the system consisting of two noninteracting two-level atoms, each atom is trapped inside a single-mode optical cavity which is filled with a nonlinear Kerr-like medium (see Fig. 1). The Hamiltonian of the whole system can be written as [22]:

$$
\begin{aligned}
H & =\sum_{i=1}^{2}\left[\omega_{\mathrm{c}} a_{i}^{\dagger} a_{i}+\frac{\omega_{a}}{2} \sigma_{i}^{z}+\chi a_{i}^{\dagger 2} a_{i}^{2}\right. \\
& \left.+g\left(a_{i}^{\dagger 2} \sigma_{i}^{-}+a_{i}^{2} \sigma_{i}^{+}\right)\right]
\end{aligned}
$$

where $a^{\dagger}$ and $a$ are the creation and annihilation operators of the cavity mode, $\sigma^{z}$ and $\sigma^{ \pm}$are the atomic operators, $\omega_{a}$ is the atomic transition frequency, $g$ is atom-field coupling constant and $\chi$ is the Kerr coupling constant. For simplicity, we consider the resonance case and set $\hbar=1$ in this paper.

For a bipartite system, there are some effective and equivalent methods for measuring of the entanglement, such as log-negativity, entanglement entropy, concurrence and so on. In this paper, we adopt the concurrence [23] to quantify the degree of entanglement. For two qubits, the concurrence can be defined as 


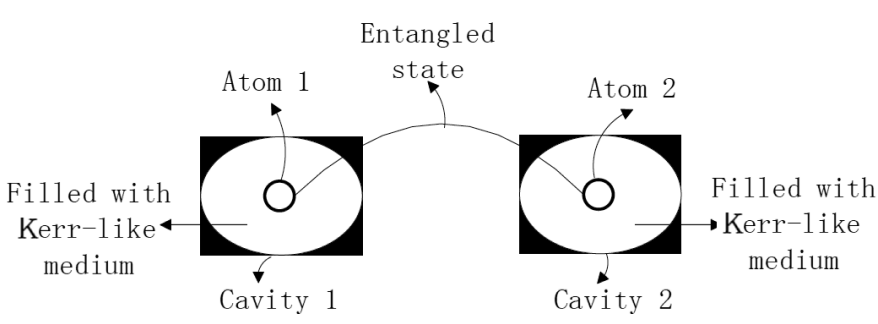

Fig. 1. Schematic diagram of the system which is investigated in the present paper.

$$
C(\rho)=\max \left\{0, \sqrt{\lambda_{1}}-\sqrt{\lambda_{2}}-\sqrt{\lambda_{3}}-\sqrt{\lambda_{4}}\right\},
$$

where the $\lambda_{i}(i=1,2,3,4)$ are the eigenvalues in a decreasing order of the magnitude of the "spin-flipped" density matrix operator $R=\rho\left(\sigma_{y} \otimes \sigma_{y}\right) \rho^{*}\left(\sigma_{y} \otimes \sigma_{y}\right)$ and $\sigma_{y}$ is the Pauli $Y$ matrix, $\rho^{*}$ is the complex conjugate of $\rho$. Particularly, for a density matrix of the form

$$
\boldsymbol{\rho}=\left(\begin{array}{cccc}
a & 0 & 0 & f \\
0 & b & e & 0 \\
0 & \mathrm{e}^{*} & c & 0 \\
f^{*} & 0 & 0 & d
\end{array}\right),
$$

called " $X$ states", where $a+b+c+d=1$. The concurrence can be easily computed as [24]:

$$
C(\rho)=2 \max \{0,|e|-\sqrt{a d},|f|-\sqrt{b c}\} .
$$

Next, we study the influence of the nonlinear Kerr-like medium on the entanglement sudden death and sudden birth in this system. We assume the initial states of the two atoms are prepared in the extended Werner-like states and the two cavities are prepared in vacuum states. The extended Werner-like states are defined by [25]:

$$
\begin{aligned}
& \rho_{\Phi}(0)=r|\Phi\rangle\langle\Phi|+\frac{1-r}{4} I, \\
& \rho_{\Psi}(0)=r|\Psi\rangle\langle\Psi|+\frac{1-r}{4} I, \\
& |\Phi\rangle=\mu|g e\rangle+\nu|e g\rangle, \\
& |\Psi\rangle=\mu|g g\rangle+\nu|e e\rangle,
\end{aligned}
$$

where $r$ is a real number which indicates the purity of initial states, for $r=0$ the extended Werner-like states become totally mixed states, while for $r=1$ they are the well-known Bell states. $I$ is a $4 \times 4$ identity matrix, $\mu$ and $\nu$ are complex numbers with $|\mu|^{2}+|\nu|^{2}=1,|e\rangle$ and $|g\rangle$ are the excited and ground states of the two-level atom.

We first consider that the density matrix of the whole system at $t=0$ is

$$
\rho(0)=\rho_{\Phi}(0) \otimes|0\rangle_{c_{1} c_{1}}\langle 0|\otimes| 0\rangle_{c_{2} c_{2}}\langle 0| .
$$

It is not difficult to find that the density matrix of the system at time $t$ is

$$
\begin{aligned}
& \rho(t)=\frac{1-r}{4}\left[A_{0}^{2}(t)|e e\rangle_{a_{1} a_{2}}|00\rangle_{c_{1} c_{2}}\right. \\
& +A_{0}(t) B_{0}(t)|e g\rangle_{a_{1} a_{2}}|02\rangle_{c_{1} c_{2}} \\
& +B_{0}(t) A_{0}(t)|g e\rangle_{a_{1} a_{2}}|20\rangle_{c_{1} c_{2}} \\
& \left.+B_{0}^{2}(t)|g g\rangle_{a_{1} a_{2}}|22\rangle_{c_{1} c_{2}}\right]\left[A _ { 0 } ^ { * 2 } ( t ) \left\langlee e | _ { a _ { 1 } a _ { 2 } } \left\langle\left.00\right|_{c_{1} c_{2}}\right.\right.\right. \\
& +A_{0}^{*}(t) B_{0}^{*}(t)\left\langlee g | _ { a _ { 1 } a _ { 2 } } \left\langle\left. 02\right|_{c_{1} c_{2}}\right.\right. \\
& +B_{0}^{*}(t) A_{0}^{*}(t)\left\langleg e | _ { a _ { 1 } a _ { 2 } } \left\langle\left. 20\right|_{c_{1} c_{2}}\right.\right. \\
& +B_{0}^{* 2}(t)\left\langle\left. g g\right|_{a_{1} a_{2}}\left\langle\left. 22\right|_{c_{1} c_{2}}\right]\right. \\
& +\left(r|\nu|^{2}+\frac{1-r}{4}\right)\left[A_{0}(t)|e g\rangle_{a_{1} a_{2}}|00\rangle_{c_{1} c_{2}}\right. \\
& \left.+B_{0}(t)|g g\rangle_{a_{1} a_{2}}|20\rangle_{c_{1} c_{2}}\right]\left[A _ { 0 } ^ { * } ( t ) \left\langlee g | _ { a _ { 1 } a _ { 2 } } \left\langle\left.00\right|_{c_{1} c_{2}}\right.\right.\right. \\
& +B_{0}^{*}(t)\left\langle\left. g g\right|_{a_{1} a_{2}}\left\langle\left. 20\right|_{c_{1} c_{2}}\right]\right. \\
& +r \nu \mu^{*}\left[A_{0}(t)|e g\rangle_{a_{1} a_{2}}|00\rangle_{c_{1} c_{2}}\right. \\
& \left.+B_{0}(t)|g g\rangle_{a_{1} a_{2}}|02\rangle_{c_{1} c_{2}}\right]\left[A _ { 0 } ^ { * } ( t ) \left\langleg e | _ { a _ { 1 } a _ { 2 } } \left\langle\left.00\right|_{c_{1} c_{2}}\right.\right.\right. \\
& +B_{0}^{*}(t)\left\langle\left. g g\right|_{a_{1} a_{2}}\left\langle\left. 02\right|_{c_{1} c_{2}}\right]\right. \\
& +r \mu \nu^{*}\left[A_{0}(t)|g e\rangle_{a_{1} a_{2}}|00\rangle_{c_{1} c_{2}}\right. \\
& \left.+B_{0}(t)|g g\rangle_{a_{1} a_{2}}|02\rangle_{c_{1} c_{2}}\right]\left[A _ { 0 } ^ { * } ( t ) \left\langlee g | _ { a _ { 1 } a _ { 2 } } \left\langle\left.00\right|_{c_{1} c_{2}}\right.\right.\right. \\
& +B_{0}^{*}(t)\left\langle\left. g g\right|_{a_{1} a_{2}}\left\langle\left. 20\right|_{c_{1} c_{2}}\right]\right. \\
& +\left(r|\mu|^{2}+\frac{1-r}{4}\right)\left[A_{0}(t)|g e\rangle_{a_{1} a_{2}}|00\rangle_{c_{1} c_{2}}\right. \\
& \left.+B_{0}(t)|g g\rangle_{a_{1} a_{2}}|02\rangle_{c_{1} c_{2}}\right]\left[A _ { 0 } ^ { * } ( t ) \left\langleg e | _ { a _ { 1 } a _ { 2 } } \left\langle\left.00\right|_{c_{1} c_{2}}\right.\right.\right. \\
& +B_{0}^{*}(t)\left\langle\left. g g\right|_{a_{1} a_{2}}\left\langle\left. 02\right|_{c_{1} c_{2}}\right]\right. \\
& +\frac{1-r}{4}|g g\rangle_{a_{1} a_{2}}|00\rangle_{c_{1} c_{2}}\left\langleg g | _ { a _ { 1 } a _ { 2 } } \left\langle\left. 00\right|_{c_{1} c_{2}},\right.\right.
\end{aligned}
$$

with

$$
\begin{aligned}
& A_{0}(t)=\exp (-\mathrm{i} \chi t)\left[\cos (\Omega t)+\frac{\mathrm{i} \chi}{\Omega} \sin (\Omega t)\right], \\
& B_{0}(t)=\exp (-\mathrm{i} \chi t)\left[\frac{-\mathrm{i} \sqrt{2} g \sin (\Omega t)}{\Omega}\right],
\end{aligned}
$$

where $\Omega=\sqrt{2 g^{2}+\chi^{2}}$.

Tracing over the degrees of the freedom of cavities, we obtain the reduced density matrix of atoms $a_{1}$ and $a_{2}$ :

$$
\begin{aligned}
& \rho_{a_{1} a_{2}}(t)=\frac{1-r}{4}\left[| A _ { 0 } ^ { 2 } ( t ) | ^ { 2 } | e e \rangle _ { a _ { 1 } a _ { 2 } } \left\langle\left.e e\right|_{a_{1} a_{2}}\right.\right. \\
& +\left|A_{0}(t) B_{0}(t)\right|^{2}|e g\rangle_{a_{1} a_{2}}\left\langle\left. e g\right|_{a_{1} a_{2}}\right. \\
& +\left|A_{0}(t) B_{0}(t)\right|^{2}|g e\rangle_{a_{1} a_{2}}\left\langle\left. g e\right|_{a_{1} a_{2}}\right.
\end{aligned}
$$




$$
\begin{aligned}
& +\left|B_{0}^{2}(t)\right|^{2}|g g\rangle_{a_{1} a_{2}}\left\langle\left. g g\right|_{a_{1} a_{2}}\right] \\
& +\left(r|\nu|^{2}+\frac{1-r}{4}\right)\left[| A _ { 0 } ( t ) | ^ { 2 } | e g \rangle _ { a _ { 1 } a _ { 2 } } \left\langle\left.e g\right|_{a_{1} a_{2}}\right.\right. \\
& +\left|B_{0}(t)\right|^{2}|g g\rangle_{a_{1} a_{2}}\left\langle\left. g g\right|_{a_{1} a_{2}}\right] \\
& +r \nu \mu^{*}\left[\left|A_{0}(t)\right|^{2}|e g\rangle_{a_{1} a_{2}}\left\langle\left. g e\right|_{a_{1} a_{2}}\right]\right. \\
& +r \mu \nu^{*}\left[\left|A_{0}(t)\right|^{2}|g e\rangle_{a_{1} a_{2}}\left\langle\left. e g\right|_{a_{1} a_{2}}\right]\right. \\
& +\left(r|\mu|^{2}+\frac{1-r}{4}\right)\left[| A _ { 0 } ( t ) | ^ { 2 } | g e \rangle _ { a _ { 1 } a _ { 2 } } \left\langle\left.g e\right|_{a_{1} a_{2}}\right.\right. \\
& +\left|B_{0}(t)\right|^{2}|g g\rangle_{a_{1} a_{2}}\left\langle\left. g g\right|_{a_{1} a_{2}}\right] \\
& +\frac{1-r}{4}|g g\rangle_{a_{1} a_{2}}\left\langle\left. g g\right|_{a_{1} a_{2}},\right.
\end{aligned}
$$

which leads to the explicit expression of concurrence of the above density matrix,

$$
\begin{gathered}
C_{a_{1} a_{2}}(t)=2 \max \left\{0,|r \mu \nu|\left|A_{0}(t)\right|^{2}-\sqrt{\frac{1-r}{4}}\left|A_{0}(t)\right|^{2}\right. \\
\times\left[\frac{1-r}{4}\left|B_{0}^{2}(t)\right|^{2}+\left(r|\nu|^{2}+\frac{1-r}{4}\right)\left|B_{0}(t)\right|^{2}\right. \\
\left.\left.+\left(r|\mu|^{2}+\frac{1-r}{4}\right)\left|B_{0}(t)\right|^{2}+\frac{1-r}{4}\right]^{1 / 2}\right\}
\end{gathered}
$$

Similarly, the reduced density matrix of two cavities can be calculated as

$$
\begin{aligned}
& \rho_{c_{1} c_{2}}(t)=\frac{1-r}{4}\left[| A _ { 0 } ^ { 2 } ( t ) | ^ { 2 } | 0 0 \rangle _ { c _ { 1 } c _ { 2 } } \left\langle\left.00\right|_{c_{1} c_{2}}\right.\right. \\
& \quad+\left|A_{0}(t) B_{0}(t)\right|^{2}|02\rangle_{c_{1} c_{2}}\left\langle\left. 02\right|_{c_{1} c_{2}}\right. \\
& \quad+\left|A_{0}(t) B_{0}(t)\right|^{2}|20\rangle_{c_{1} c_{2}}\left\langle\left. 20\right|_{c_{1} c_{2}}\right. \\
& \quad+\left|B_{0}^{2}(t)\right|^{2}|22\rangle_{c_{1} c_{2}}\left\langle\left. 22\right|_{c_{1} c_{2}}\right] \\
& \quad+\left(r|\nu|^{2}+\frac{1-r}{4}\right)\left[| A _ { 0 } ( t ) | ^ { 2 } | 0 0 \rangle _ { c _ { 1 } c _ { 2 } } \left\langle\left.00\right|_{c_{1} c_{2}}\right.\right. \\
& \quad+\left|B_{0}(t)\right|^{2}|20\rangle_{c_{1} c_{2}}\left\langle\left. 20\right|_{c_{1} c_{2}}\right] \\
& \quad+r \nu \mu^{*}\left[\left|B_{0}(t)\right|^{2}|20\rangle_{c_{1} c_{2}}\left\langle\left. 02\right|_{c_{1} c_{2}}\right]\right. \\
& \quad+r \mu \nu^{*}\left[\left|B_{0}(t)\right|^{2}|02\rangle_{c_{1} c_{2}}\left\langle\left. 20\right|_{c_{1} c_{2}}\right]\right. \\
& \quad+\left(r|\mu|^{2}+\frac{1-r}{4}\right)\left[| A _ { 0 } ( t ) | ^ { 2 } | 0 0 \rangle _ { c _ { 1 } c _ { 2 } } \left\langle\left.00\right|_{c_{1} c_{2}}\right.\right. \\
& +\left|B_{0}(t)\right|^{2}|02\rangle_{c_{1} c_{2}}\left\langle\left. 02\right|_{c_{1} c_{2}}\right] \\
& +\frac{1-r}{4}|00\rangle_{c_{1} c_{2}}\left\langle\left. 00\right|_{c_{1} c_{2}},\right.
\end{aligned}
$$

and the concurrence is given by

$$
C_{c_{1} c_{2}}(t)=2 \max \left\{0,\left|r \mu \nu \| B_{0}(t)\right|^{2}-\sqrt{\frac{1-r}{4}}\left|B_{0}(t)\right|^{2}\right.
$$

$$
\begin{aligned}
& \times\left[\frac{1-r}{4}\left|A_{0}^{2}(t)\right|^{2}+\left(r|\nu|^{2}+\frac{1-r}{4}\right)\left|A_{0}(t)\right|^{2}\right. \\
& \left.\left.+\left(r|\mu|^{2}+\frac{1-r}{4}\right)\left|A_{0}(t)\right|^{2}+\frac{1-r}{4}\right]^{1 / 2}\right\} .
\end{aligned}
$$

In Fig. 2, we plot the evolution of concurrence between the two atoms $C_{a_{1} a_{2}}$ (solid line) and the two cavities $C_{c_{1} c_{2}}$ (dotted line) for different values of the Kerr coefficient $\chi$ with $r=2 / 3,|\mu|^{2}=1 / 12,|\nu|^{2}=11 / 12$ and $g=1$. We can see that the concurrence $C_{a_{1} a_{2}}$ disappears suddenly and the concurrence $C_{c_{1} c_{2}}$ appears suddenly during the dynamics evolution, which means that the ESD (solid line) and ESB (dotted line) appears in this system. The ESD and ESB phenomenon demonstrate that the entanglement contained initially in the atom-atom subsystem is transferred to the cavity-cavity subsystem. By requiring that $C_{a 1 a 2}\left(t_{\mathrm{ESD}}\right)=0$ and $C_{c 1 c 2}\left(t_{\mathrm{ESB}}\right)=0$ in Eqs. (10) and (12), respectively, we can obtain the times for which ESD and ESB occur

$$
\begin{aligned}
& t_{\mathrm{ESD}}=\frac{1}{\Omega} \arcsin \left(\frac{\Omega}{g} \sqrt{\frac{16 r^{2}|\mu \nu|^{2}-(1-r)^{2}}{2(1-r)(3+r)}}\right), \\
& t_{\mathrm{ESB}}=\frac{1}{\Omega} \arcsin \left(\frac{\Omega}{g} \sqrt{\frac{2\left(1-r-4 r^{2}|\mu \nu|^{2}\right)}{(1-r)(3+r)}}\right),
\end{aligned}
$$

where $\Omega=\sqrt{2 g^{2}+\chi^{2}}$. From the above formulae, it is not difficult to find that the times for which ESD $\left(t_{\mathrm{ESD}}\right)$ and $\mathrm{ESB}\left(t_{\mathrm{ESB}}\right)$ occur and the amount of entanglement between two cavities could be altered by adjusting the Kerr coefficient $\chi$ and the parameters of the initial states. Furthermore, from the above relations one can learn that the phenomenon of ESD occurs for $(1+4|\mu \nu|)^{-1}<r<\left(\sqrt{1+16|\mu \nu|^{2}}-1\right) /\left(8|\mu \nu|^{2}\right)$ in the case of the Kerr coefficient $\chi=0$.

In Fig. 3, we plot the evolution of two-qubit concurrence $C_{a_{1} a_{2}}$ (solid line) and $C_{c_{1} c_{2}}$ (dotted line) for different values of the Kerr coefficient $\chi$ with $r=2 / 3$, $|\mu|^{2}=1 / 6,|\nu|^{2}=5 / 6$ and $g=1$. Comparing with Fig. 2, we can see the times for which ESD $\left(t_{\mathrm{ESD}}\right)$ and $\mathrm{ESB}\left(t_{\mathrm{ESB}}\right)$ occur depending on the parameters $\mu$ and $\nu$. In the case of $|\mu|^{2}=1 / 12$ and $|\nu|^{2}=11 / 12, t_{\mathrm{ESD}}<t_{\mathrm{ESB}}$, namely, ESD appears before ESB. Nevertheless when $|\mu|^{2}=1 / 6$ and $|\nu|^{2}=5 / 6, t_{\mathrm{ESD}}>t_{\mathrm{ESB}}$, namely, ESD appears after ESB. Again, the duration of ESD and ESB and the amount of entanglement between two cavities can be altered by adjusting the Kerr coefficient $\chi$. Particularly, from the part (c) of Fig. 2 and Fig. 3, we can find that the phenomenon of ESD and ESB disappears one after another if we increase the value of the Kerr coefficient $\chi$ continuously. In the part (c) of Fig. 2, the ESB disappears earlier than ESD. But in the part (c) of Fig. 3, the ESD disappears earlier than ESB. This means that the phenomenon of the ESD and ESB can be controlled by adjusting the Kerr coefficient $\chi$ and disappears one after another. The phenomenon of ESD and ESB all disappears if the value of the Kerr coefficient $\chi$ is large enough. The physical explain is that in the case of $g \ll \chi$, 


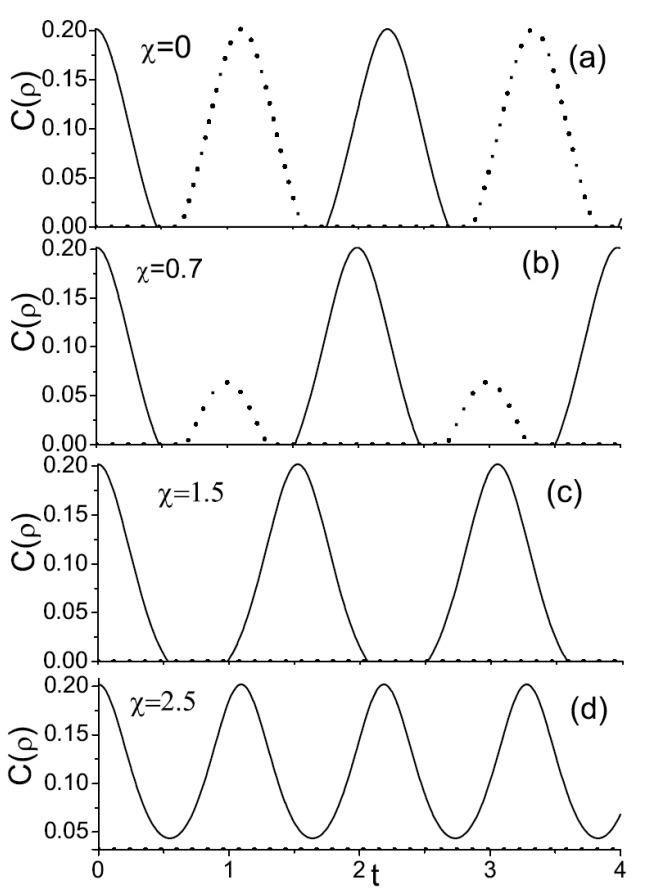

Fig. 2. Evolution of two-qubit concurrence $C_{a_{1} a_{2}}$ (solid line) and $C_{c_{1} c_{2}}$ (dotted line), for the initial state of atoms $\rho_{\Phi}(0)$ with $r=2 / 3,|\mu|^{2}=1 / 12,|\nu|^{2}=11 / 12$ and $g=1$. (a) $\chi=0$; (b) $\chi=0.7$; (c) $\chi=1.5$; (d) $\chi=2.5$.

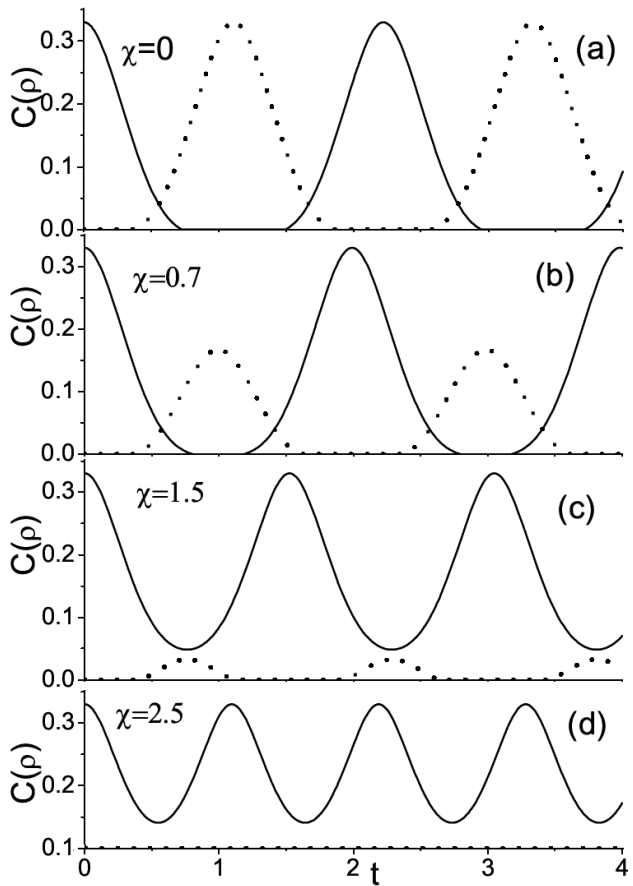

Fig. 3. Evolution of two-qubit concurrence $C_{a_{1} a_{2}}$ (solid line) and $C_{c_{1} c_{2}}$ (dotted line), for the initial state of atoms $\rho_{\Phi}(0)$ with $r=2 / 3,|\mu|^{2}=1 / 6,|\nu|^{2}=5 / 6$ and $g=1$. (a) $\chi=0$; (b) $\chi=0.7$; (c) $\chi=1.5$; (d) $\chi=2.5$. the $A_{0}(t)$ and $B_{0}(t)$ approximately behave as when $g=0$, which refers to the situation in which the atoms would not interact with the cavities. Of course, without interaction no flow of entanglement can occur among the different partitions and hence no ESD and ESB.

In the following, we assume that the initial states of the two atoms are in the $\rho_{\Psi}(0)$ and the density matrix of the system at $t=0$ reads

$$
\rho(0)=\rho_{\Psi}(0) \otimes|0\rangle_{c_{1} c_{1}}\langle 0|\otimes| 0\rangle_{c_{2} c_{2}}\langle 0| \text {. }
$$

A similar calculation shows that the density matrix of the system at time $t$ is

$$
\begin{aligned}
& \rho(t)=\left(r|\nu|^{2}+\frac{1-r}{4}\right)\left[A_{0}^{2}(t)|e e\rangle_{a_{1} a_{2}}|00\rangle_{c_{1} c_{2}}\right. \\
& +A_{0}(t) B_{0}(t)|e g\rangle_{a_{1} a_{2}}|02\rangle_{c_{1} c_{2}} \\
& +B_{0}(t) A_{0}(t)|g e\rangle_{a_{1} a_{2}}|20\rangle_{c_{1} c_{2}} \\
& \left.+B_{0}^{2}(t)|g g\rangle_{a_{1} a_{2}}|22\rangle_{c_{1} c_{2}}\right]\left[A _ { 0 } ^ { * 2 } ( t ) \left\langlee e | _ { a _ { 1 } a _ { 2 } } \left\langle\left.00\right|_{c_{1} c_{2}}\right.\right.\right. \\
& +A_{0}^{*}(t) B_{0}^{*}(t)\left\langlee g | _ { a _ { 1 } a _ { 2 } } \left\langle\left. 02\right|_{c_{1} c_{2}}\right.\right. \\
& +B_{0}^{*}(t) A_{0}^{*}(t)\left\langleg e | _ { a _ { 1 } a _ { 2 } } \left\langle\left. 20\right|_{c_{1} c_{2}}\right.\right. \\
& +B_{0}^{* 2}(t)\left\langle\left. g g\right|_{a_{1} a_{2}}\left\langle\left. 22\right|_{c_{1} c_{2}}\right]\right. \\
& +\frac{1-r}{4}\left[A_{0}(t)|e g\rangle_{a_{1} a_{2}}|00\rangle_{c_{1} c_{2}}\right. \\
& \left.+B_{0}(t)|g g\rangle_{a_{1} a_{2}}|20\rangle_{c_{1} c_{2}}\right]\left[A _ { 0 } ^ { * } ( t ) \left\langlee g | _ { a _ { 1 } a _ { 2 } } \left\langle\left.00\right|_{c_{1} c_{2}}\right.\right.\right. \\
& +B_{0}^{*}(t)\left\langle\left. g g\right|_{a_{1} a_{2}}\left\langle\left. 20\right|_{c_{1} c_{2}}\right]\right. \\
& +\frac{1-r}{4}\left[A_{0}(t)|g e\rangle_{a_{1} a_{2}}|00\rangle_{c_{1} c_{2}}\right. \\
& \left.+B_{0}(t)|g g\rangle_{a_{1} a_{2}}|02\rangle_{c_{1} c_{2}}\right]\left[A _ { 0 } ^ { * } ( t ) \left\langleg e | _ { a _ { 1 } a _ { 2 } } \left\langle\left.00\right|_{c_{1} c_{2}}\right.\right.\right. \\
& +B_{0}^{*}(t)\left\langle\left. g g\right|_{a_{1} a_{2}}\left\langle\left. 02\right|_{c_{1} c_{2}}\right]\right. \\
& +\left(r|\mu|^{2}+\frac{1-r}{4}\right)|g g\rangle_{a_{1} a_{2}}|00\rangle_{c_{1} c_{2}}\left\langleg g | _ { a _ { 1 } a _ { 2 } } \left\langle\left. 00\right|_{c_{1} c_{2}}\right.\right. \\
& +r \nu \mu^{*}\left[A_{0}^{2}(t)|e e\rangle_{a_{1} a_{2}}|00\rangle_{c_{1} c_{2}}\right. \\
& +A_{0}(t) B_{0}(t)|e g\rangle_{a_{1} a_{2}}|02\rangle_{c_{1} c_{2}} \\
& +B_{0}(t) A_{0}(t)|g e\rangle_{a_{1} a_{2}}|20\rangle_{c_{1} c_{2}} \\
& \left.+B_{0}^{2}(t)|g g\rangle_{a_{1} a_{2}}|22\rangle_{c_{1} c_{2}}\right]\left\langleg g | _ { a _ { 1 } a _ { 2 } } \left\langle\left. 00\right|_{c_{1} c_{2}}\right.\right. \\
& +r \mu \nu^{*}|g g\rangle_{a_{1} a_{2}}|00\rangle_{c_{1} c_{2}}\left[A _ { 0 } ^ { * 2 } ( t ) \left\langlee e | _ { a _ { 1 } a _ { 2 } } \left\langle\left.00\right|_{c_{1} c_{2}}\right.\right.\right. \\
& +A_{0}^{*}(t) B_{0}^{*}(t)\left\langlee g | _ { a _ { 1 } a _ { 2 } } \left\langle\left. 02\right|_{c_{1} c_{2}}\right.\right. \\
& +B_{0}^{*}(t) A_{0}^{*}(t)\left\langleg e | _ { a _ { 1 } a _ { 2 } } \left\langle\left. 20\right|_{c_{1} c_{2}}\right.\right. \\
& +B_{0}^{* 2}(t)\left\langle\left. g g\right|_{a_{1} a_{2}}\left\langle\left. 22\right|_{c_{1} c_{2}}\right] .\right.
\end{aligned}
$$


Tracing over the degrees of the freedom of cavities, we obtain the reduced density matrix of atoms $a_{1}$ and $a_{2}$,

$$
\begin{aligned}
& \rho_{a_{1} a_{2}}(t)=\left(r|\nu|^{2}+\frac{1-r}{4}\right)\left[| A _ { 0 } ^ { 2 } ( t ) | ^ { 2 } | e e \rangle _ { a _ { 1 } a _ { 2 } } \left\langle\left.e e\right|_{a_{1} a_{2}}\right.\right. \\
& +\left|A_{0}(t) B_{0}(t)\right|^{2}|e g\rangle_{a_{1} a_{2}}\left\langle\left. e g\right|_{a_{1} a_{2}}\right. \\
& \quad+\left|A_{0}(t) B_{0}(t)\right|^{2}|g e\rangle_{a_{1} a_{2}}\left\langle\left. g e\right|_{a_{1} a_{2}}\right. \\
& +\left|B_{0}^{2}(t)\right|^{2}|g g\rangle_{a_{1} a_{2}}\left\langle\left. g g\right|_{a_{1} a_{2}}\right] \\
& \quad+\frac{1-r}{4}\left[| A _ { 0 } ( t ) | ^ { 2 } | e g \rangle _ { a _ { 1 } a _ { 2 } } \left\langle\left.e g\right|_{a_{1} a_{2}}\right.\right. \\
& \quad+\left|B_{0}(t)\right|^{2}|g g\rangle_{a_{1} a_{2}}\left\langle\left. g g\right|_{a_{1} a_{2}}\right] \\
& +\frac{1-r}{4}\left[| A _ { 0 } ( t ) | ^ { 2 } | g e \rangle _ { a _ { 1 } a _ { 2 } } \left\langle\left.g e\right|_{a_{1} a_{2}}\right.\right. \\
& +\left|B_{0}(t)\right|^{2}|g g\rangle_{a_{1} a_{2}}\left\langle\left. g g\right|_{a_{1} a_{2}}\right] \\
& \quad+\left(r|\mu|^{2}+\frac{1-r}{4}\right)|g g\rangle_{a_{1} a_{2}}\left\langle\left. g g\right|_{a_{1} a_{2}}\right. \\
& \quad+r \nu \mu^{*}\left[A_{0}^{2}(t)|e e\rangle_{a_{1} a_{2}}\left\langle\left. g g\right|_{a_{1} a_{2}}\right]\right. \\
& +r \mu \nu^{*}\left[A_{0}^{* 2}(t)|g g\rangle_{a_{1} a_{2}}\left\langle\left. e e\right|_{a_{1} a_{2}}\right] .\right.
\end{aligned}
$$

Inserting Eq. (16) into Eq. (4), we find that the explicit expression of the concurrence between two atoms is

$$
\begin{gathered}
C_{a_{1} a_{2}}(t)=2 \max \left\{0,|r \mu \nu|\left|A_{0}(t)\right|^{2}-\left[\left(r|\nu|^{2}+\frac{1-r}{4}\right)\right.\right. \\
\left.\left.\times\left|B_{0}(t) A_{0}(t)\right|^{2}+\frac{1-r}{4}\left|A_{0}(t)\right|^{2}\right]\right\} .
\end{gathered}
$$

Similarly, the reduced density matrix of two cavities is

$$
\begin{aligned}
& \rho_{c_{1} c_{2}}(t)=\left(r|\nu|^{2}+\frac{1-r}{4}\right)\left[| A _ { 0 } ^ { 2 } ( t ) | ^ { 2 } | 0 0 \rangle _ { c _ { 1 } c _ { 2 } } \left\langle\left.00\right|_{c_{1} c_{2}}\right.\right. \\
& +\left|A_{0}(t) B_{0}(t)\right|^{2}|02\rangle_{c_{1} c_{2}}\left\langle\left. 02\right|_{c_{1} c_{2}}\right. \\
& +\left|A_{0}(t) B_{0}(t)\right|^{2}|20\rangle_{c_{1} c_{2}}\left\langle\left. 20\right|_{c_{1} c_{2}}\right. \\
& +\left|B_{0}^{2}(t)\right|^{2}|22\rangle_{c_{1} c_{2}}\left\langle\left. 22\right|_{c_{1} c_{2}}\right] \\
& \quad+\frac{1-r}{4}\left[| A _ { 0 } ( t ) | ^ { 2 } | 0 0 \rangle _ { c _ { 1 } c _ { 2 } } \left\langle\left.00\right|_{c_{1} c_{2}}\right.\right. \\
& \quad+\left|B_{0}(t)\right|^{2}|20\rangle_{c_{1} c_{2}}\left\langle\left. 20\right|_{c_{1} c_{2}}\right] \\
& \quad+\frac{1-r}{4}\left[| A _ { 0 } ( t ) | ^ { 2 } | 0 0 \rangle _ { c _ { 1 } c _ { 2 } } \left\langle\left.00\right|_{c_{1} c_{2}}\right.\right. \\
& +\left|B_{0}(t)\right|^{2}|02\rangle_{c_{1} c_{2}}\left\langle\left. 02\right|_{c_{1} c_{2}}\right] \\
& \quad+\left(r|\mu|^{2}+\frac{1-r}{4}\right)|00\rangle_{c_{1} c_{2}}\left\langle\left. 00\right|_{c_{1} c_{2}}\right. \\
& +r \nu \mu^{*} B_{0}^{2}(t)|22\rangle_{c_{1} c_{2}}\left\langle\left. 00\right|_{c_{1} c_{2}}\right.
\end{aligned}
$$

$$
+r \mu \nu^{*} B_{0}^{* 2}(t)|00\rangle_{c_{1} c_{2}}\left\langle\left. 22\right|_{c_{1} c_{2}},\right.
$$

and the concurrence is

$$
\begin{gathered}
C_{c_{1} c_{2}}(t)=2 \max \left\{0,|r \mu \nu|\left|B_{0}(t)\right|^{2}-\left[\left(r|\nu|^{2}+\frac{1-r}{4}\right)\right.\right. \\
\left.\left.\times\left|B_{0}(t) A_{0}(t)\right|^{2}+\frac{1-r}{4}\left|B_{0}(t)\right|^{2}\right]\right\} .
\end{gathered}
$$

In Fig. 4, we plot the evolution of concurrence between the two atoms $C_{a_{1} a_{2}}$ (solid line) and the two cavities $C_{c_{1} c_{2}}$ (dotted line) for different values of the Kerr coefficient $\chi$ with $r=2 / 3,|\mu|^{2}=1 / 6,|\nu|^{2}=5 / 6$ and $g=1$. On the one hand, we find that the concurrence of two atoms $C_{a_{1} a_{2}}$ disappears within a finite time during the dynamics evolution (solid line). On the other hand, the concurrence of two cavities $C_{c_{1} c_{2}}$ appears during the dynamics evolution (dotted line), which means that the entanglement contained initially in the atomatom subsystem flows into the cavity-cavity subsystem. By requiring that $C_{a_{1} a_{2}}\left(t_{\mathrm{ESD}}\right)=0$ and $C_{c_{1} c_{2}}\left(t_{\mathrm{ESB}}\right)=0$ in Eqs. (17) and (19), respectively, we can find the times for which ESD and ESB occur

$$
\begin{aligned}
& t_{\mathrm{ESD}}=\frac{1}{\Omega} \arccos \left(\sqrt{1-\frac{\Omega^{2}}{2 g^{2}} \frac{(4 r|\mu \nu|+r-1)}{\left(4 r|\nu|^{2}-r+1\right)}}\right), \\
& t_{\mathrm{ESB}}=\frac{1}{\Omega} \arccos \left(\sqrt{-\frac{\chi^{2}}{2 g^{2}}+\frac{\Omega^{2}}{2 g^{2}} \frac{(4 r|\mu \nu|+r-1)}{\left(4 r|\nu|^{2}-r+1\right)}}\right),
\end{aligned}
$$

where $\Omega=\sqrt{2 g^{2}+\chi^{2}}$. From the above expressions, it is not difficult to find that the times for which $\operatorname{ESD}\left(t_{\mathrm{ESD}}\right)$ and $\operatorname{ESB}\left(t_{\mathrm{ESB}}\right)$ occur and the amount of entanglement between two cavities could be altered by adjusting the Kerr coefficient $\chi$ and the parameters of the initial states. Furthermore, one can see that the phenomenon of ESD occurs if we choose the parameters of the initial states to satisfy two inequalities $\left(2|\mu \nu|+1-2|\nu|^{2}\right) r<1$ and $r>1 /(4|\mu \nu|+1)$ in the case of the Kerr coefficient $\chi=0$.

In Fig. 5, we plot the evolution of two-qubit concurrence $C_{a_{1} a_{2}}$ (solid line) and $C_{c_{1} c_{2}}$ (dotted line) for different values of the Kerr coefficient $\chi$ with $r=2 / 3$, $|\mu|^{2}=3 / 5,|\nu|^{2}=2 / 5$ and $g=1$. Comparing with Fig. 4 , we can see the times for which $\operatorname{ESD}\left(t_{\mathrm{ESD}}\right)$ and ESB $\left(t_{\mathrm{ESB}}\right)$ occur depending on the parameters $\mu$ and $\nu$. In the case of $|\mu|^{2}=1 / 6$ and $|\nu|^{2}=5 / 6$, the ESD appears before ESB, that is, $t_{\mathrm{ESD}}<t_{\mathrm{ESB}}$. However, when $|\mu|^{2}=3 / 5$ and $|\nu|^{2}=2 / 5$, the ESD appears after ESB, that is, $t_{\mathrm{ESD}}>t_{\mathrm{ESB}}$. Again, the duration of ESD and ESB and the amount of entanglement between two cavities can be altered by adjusting the Kerr coefficient $\chi$. Particularly, from part (c) of Fig. 4 and Fig. 5, we can see that the phenomenon of ESD and ESB disappears one after another if we increase the value of the Kerr coefficient $\chi$ continuously. In the part (c) of Fig. 4, the ESB disappears earlier than ESD. But in the part (c) of Fig. 5, the ESD disappears earlier than ESB. These results demonstrate that the phenomenon of the ESD and 


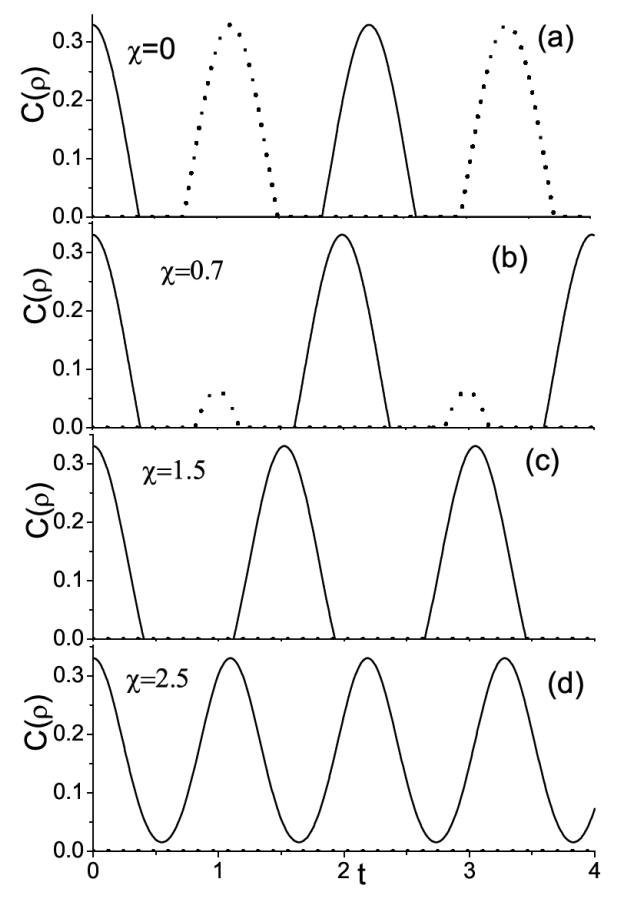

Fig. 4. Evolution of two-qubit concurrence $C_{a_{1} a_{2}}$ (solid line) and $C_{c_{1} c_{2}}$ (dotted line), for the initial state of atoms $\rho_{\Psi}(0)$ with $r=2 / 3,|\mu|^{2}=1 / 6,|\nu|^{2}=5 / 6$ and $g=1$. (a) $\chi=0$; (b) $\chi=0.7$; (c) $\chi=1.5$; (d) $\chi=2.5$.

ESB disappears one after another if we increase the value of the Kerr coefficient $\chi$ continuously, and all disappear if the value of the Kerr coefficient $\chi$ is large enough. The physical reason is similar to the case of two atoms are prepared initially in the state $\rho_{\Phi}(0)$.

In order to have an idea of how the entanglement is shared among the different partitions in the present system, we display the bipartite entanglement of $a_{1} \otimes a_{2}$, $c_{1} \otimes c_{2}, a_{1} \otimes c_{2}$, and $a_{1} \otimes c_{1}$ in Fig. $6\left(C_{(\Phi)}\right)$ and Fig. 7 $\left(C_{(\Psi)}\right)$. It can be observed that the concurrence $C_{a_{1} c_{2}}$ can exceed zero in the evolution of this system. This implies that the atom $a_{1}$ and cavity $c_{2}$ which start uncorrelated and do not interact, then become entangled as the system evolves. From Fig. 6 and Fig. 7, it is worth noting that in the region where there is no entanglement, there is, $C_{a_{1} a_{2}}=C_{c_{1} c_{2}}=C_{a_{1} c_{2}}=0$, but the entanglement between atom $a_{1}$ and its corresponding cavity $c_{1}\left(C_{a_{1} c_{1}}\right)$ reaches its maximum value. Furthermore, from Eqs. (10) and (12), we can verify that $C_{a_{1} a_{2}}(t)=2|\mu \nu| A_{0}^{2}(t)$ and $C_{c_{1} c_{2}}(t)=2|\mu \nu| B_{0}^{2}(t)$ for the initial state $\rho_{\Phi}(0)$ when $r=1$. For this case, there are no ESD and ESB, but nothing similar seems to occur for $\rho_{\Psi}(0)$. Particularly, it is clear to see that in this case $C_{a_{1} a_{2}}(t)+C_{c_{1} c_{2}}(t)=2|\mu \nu|$, which means that the amount of entanglement is conserved in these two partitions. It is interesting to check that whether the entanglement appears anywhere else in this "conservative case" in the further work.

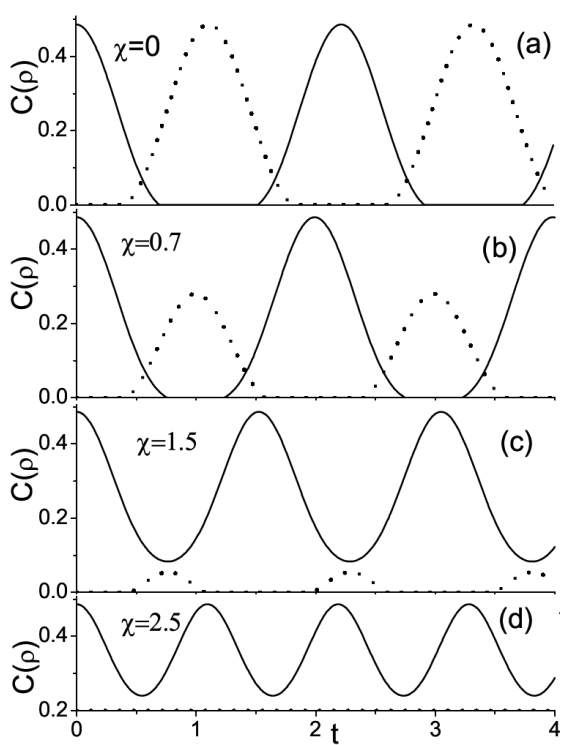

Fig. 5. Evolution of two-qubit concurrence $C_{a_{1} a_{2}}$ (solid line) and $C_{c_{1} c_{2}}$ (dotted line), for the initial state of atoms $\rho_{\Psi}(0)$ with $r=2 / 3,|\mu|^{2}=3 / 5,|\nu|^{2}=2 / 5$ and $g=1$. (a) $\chi=0$; (b) $\chi=0.7$; (c) $\chi=1.5$; (d) $\chi=2.5$.

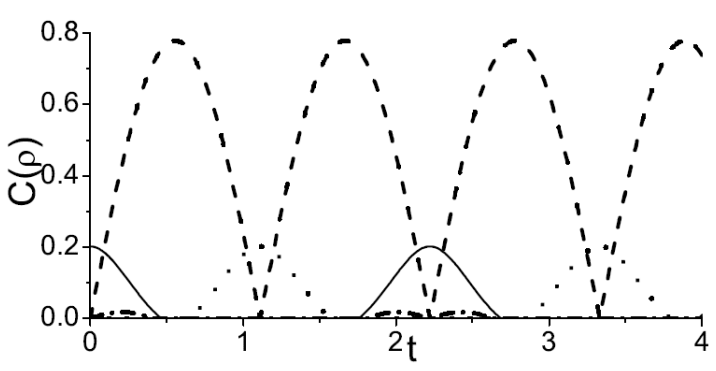

Fig. 6. Evolution of the two qubits concurrence for different bipartite partitions: $C_{a_{1} a_{2}}$ (solid line), $C_{c_{1} c_{2}}$ (dotted line), $C_{a_{1} c_{2}}$ (dot-dashed line) and $C_{a_{1} c_{1}}$ (dashed line), for the initial state of atoms $\rho_{\Phi}(0)$ with $r=2 / 3$, $g=1,|\mu|^{2}=1 / 12,|\nu|^{2}=11 / 12$ and $\chi=0$.

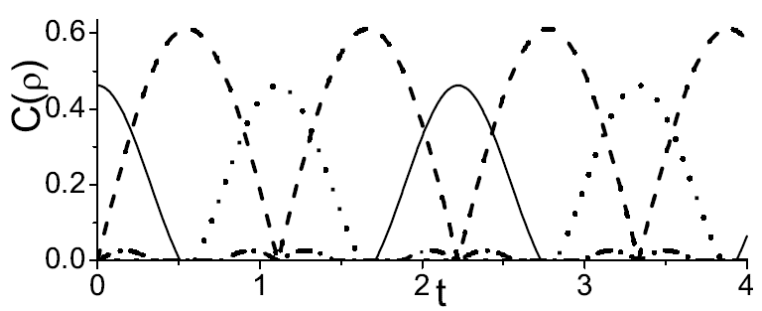

Fig. 7. Evolution of the two qubits concurrence for different bipartite partitions: $C_{a_{1} a_{2}}$ (solid line), $C_{c_{1} c_{2}}$ (dotted line), $C_{a_{1} c_{2}}$ (dot-dashed line) and $C_{a_{1} c_{1}}$ (dashed line), for the initial state of atoms $\rho_{\Psi}(0)$ with $r=2 / 3$, $g=1,|\mu|^{2}=1 / 3,|\nu|^{2}=2 / 3$ and $\chi=0$. 


\section{Conclusion}

In this paper, we investigate the entanglement dynamics of a double two-photon Jaynes-Cummings model with an added nonlinear Kerr-like medium and show that the phenomenon of ESD and ESB appears in this system. The ESD and ESB phenomenon imply that the loss of entanglement between two atoms is related to the birth of entanglement between two cavities and the other partitions. It is clear to see that ESD and ESB occur at times depending on the parameter $r, \mu$ and $\nu$ of the initial entangled states, and the ESD can occur before, together, or even after ESB. Furthermore, we find that the duration of ESD and ESB and the amount of entanglement of two qubits can be altered by adjusting the Kerr coefficient $\chi$. Particularly, the phenomenon of ESD and ESB disappears one after another if we increase the value of the Kerr coefficient $\chi$ continuously. This means that the ESD and ESB phenomenon can be controlled by adjusting the Kerr coefficient $\chi$ and disappears one after another. From the part (c) of Fig. 2 to Fig. 5, we can observe that if the ESD appears before $\operatorname{ESB}\left(t_{\mathrm{ESD}}<t_{\mathrm{ESB}}\right)$, then the ESB disappears earlier than ESD. On the contrary, the ESD disappears earlier than ESB if the ESD appears after $\mathrm{ESB}\left(t_{\mathrm{ESD}}>t_{\mathrm{ESB}}\right)$. The phenomenon of ESD and ESB all disappears if the value of the Kerr coefficient $\chi$ is large enough.

\section{Acknowledgments}

This project was supported by the National Natural Science Foundation of China (grant No. 10774131).

\section{References}

[1] J.I. Cirac, P. Zoller, Nature 404, 579 (2000).

[2] M.A. Nielsen, I.L. Chuang, Quantum Computation and Quantum Information, Cambridge University Press, Cambridge 2000.

[3] C.H. Bennett, P.W. Shor, J.A. Smolin, A.V. Thapliyal, Phys. Rev. Lett. 83, 3081 (1999).

[4] C.H. Bennett, G. Brassard, C. Crépeau, R. Jozsa, A. Peres, W.K. Wotters, Phys. Rev. Lett. 70, 1895 (1993).
[5] V. Vedral, M.B. Plenio, Phys. Rev. A 57, 1619 (1998).

[6] E. Hagley, X. Maitre, G. Nogues, C. Wunderlich, M. Brune, J.M. Raimond, S. Haroche, Phys. Rev. Lett. 79, 1 (1997).

[7] J.M. Raimond, M. Brune, S. Haroche, Rev. Mod. Phys. 73, 565 (2001).

[8] D. Braun, Phys. Rev. Lett. 89, 277901 (2002).

[9] D. Vitali, S. Gigan, A. Ferreira, H.R. Bohm, P. Tombesi, A. Guerreiro, V. Vedral, A. Zeilinger, M. Aspelmeyer, Phys. Rev. Lett. 98, 030405 (2007).

[10] T. Yu, J.H. Eberly, Phys. Rev. Lett. 93, 140404 (2004).

[11] B. Bellomo, R. Lo. Franco, G. Compagno, Phys. Rev. Lett. 99, 160502 (2007).

[12] T. Yu, J.H. Eberly, Opt. Commun. 264, 393 (2006).

[13] H.T. Cui, K. Li, X.X. Yi, Phys. Lett. A 365, 44 (2007).

[14] L. Chotorlishvili, P. Schwab, Z. Toklikishvili, V. Skrinnikov, Phys. Lett. A 374, 1642 (2010).

[15] M. Yönac, T. Yu, J.H. Eberly, J. Phys. B, At. Mol. Opt. Phys. 39, S621 (2006).

[16] J.S. Zhang, J.B. Xu, Opt. Commun. 05, 78 (2009).

[17] Q. Zheng, X.P. Zhang, Z.Z. Ren, Chin. Phys. B 17, 3553 (2008).

[18] X.C. Ouyuang, M.F. Fang, G.D. Kang, X.J. Deng, L.Y. Huang, Chin. Phys. B 19, 030309 (2010).

[19] M.P. Almeida, F. De Melo, M. Hor-Meyll, A. Salles, S.P. Walborn, P.H. Souto Ribeiro, L. Davidovich, Science 316, 579 (2007).

[20] J. Laurat, K.S. Choi, H. Deng, C.W. Chou, H.J. Kimble, Phys. Rev. Lett. 99, 180504 (2007).

[21] C.E. Lopez, G. Romero, F. Lastra, E. Solano, J.C. Retamal, Phys. Rev. Lett. 101, 080503 (2008).

[22] J. Amitabl, R.R. Puri, Phys. Rev. A 45, 5056 (1992).

[23] W.K. Wootters, Phys. Rev. Lett. 80, 2245 (1998).

[24] K.M. O'Connor, W.K. Wootters, Phys. Rev. A 63, 052302 (2001).

[25] B. Bellomo, R.Lo. France, G. Compagno, Phys. Rev. A 77, 032342 (2008). 